\title{
Two Imported Cases of New Variant COVID-19 First Emerging from Brazil — Guangdong Province, China, April 30, 2021
}

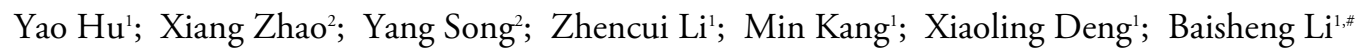

On April 15, 2021, a 62-year-old male (Patient A, XG4122) and a 58-year-old female (Patient B, XG4123) travelled from Canada via airplane and were transferred to a central isolation hotel after being tested by the laboratory of Guangzhou Customs using nose swab tests for coronavirus disease 2019 (COVID-19). The results for both patients returned COVID-19 positive and the couple were transported to the Guangzhou Eighth People's Hospital for isolated treatment by ambulance on April 16, 2021. Patient A had inflamed lungs while Patient $B$ had mild inflammation in her lungs according to the chest computed tomography (CT) examination. Both of their 28 close contacts underwent centralized isolation and medical observation.

On April 21, 2021, Guangdong Provincial CDC received the samples and began virus isolation and gene sequencing analysis. On April 27, 2021, the 2 samples were sequenced using Nanopore GridION. On April 30,2021 , the sequencing analysis concluded that the 2 virus genomes belonged to lineage P.1, 20J/501Y.V3, which was first found in four Brazilians after a routine check at Japan's Haneda Airport in late December 2020. The variant was then widely spread to Manaus in the northern State of Amazona in Brazil. The Brazil variant has been reported in 40 different countries as of April 30, 2021(1).

Compared with the Wuhan reference sequence (EPI_ISL_402119) (2-3), the strain from Patient A (XG4122) displayed 33 nucleotide variation sites (C241T, T733C, C2749T, C3037T, C3828T, A5648C, A6319G, A6613G, G9105A, C12778T, C14408T, G17259T, C21614T, C21621A, C21638T, G21974T, G22132T, A22812C, G23012A, A23063T, C23380T, A23403G, C23525T, C24642T, G25088T, T26149C, G28167A, C28512G, A28877T, G28878C, G28881A, G28882A, and G28883C) belonged to the Pangolin lineage P.1 (Figure 1). Patient B (XG4123) strain displayed 34 nucleotide variation sites (C241T, T733C, C2749T, C3037T, C3828T, A5648C, A6319G, A6613G, G9105A, C12778T, C13860T, C14408T, G17259T, C21614T, C21621A,
C21638T,
G21974T,
G22132T,
A22812C, G23012A, A23063T,
C23380T, A23403G C23525T, C24642T, G25088T, T26149C, G28167A, C28512G, A28877T, G28878C, G28881A, G28882A, and G28883C).

Furthermore, 23 amino acid mutation sites (N:P80R， N:R203K， N:G204R， ORF1a:S1188L, ORF1a:K1795Q，ORF1a:S2947N， ORF1b:P314L, ORF1b:E1264D, ORF3a:S253P, ORF8:E92K, ORF9b:Q77E, S:L18F, S:T20N, S:P26S, S:D138Y, S:R190S，S:K417T，S:E484K，S:N501Y，S:D614G, S:H655Y, S:T1027I, and S:V1176F) and 3 amino acid deletions (ORF1a:S3675-, ORF1a:G3676-, and ORF1a:F3677-) were detected in the protein that corresponded to the features of the Brazil variant (P.1) (4). Both variants have 12 mutations to the spike protein, including 3 mutations of concern in common with 20H/501Y.V2 (K417N/T, E484K, and N501Y) which may affect transmissibility and host immune response (4-5).

The P.1 lineage and the B.1.1.7 first described in the United Kingdom share the spike N501Y mutation and a deletion in ORF1b (del 3675-3677 SGF). The P.1 lineage and the B.1.351 (also known as 501Y.V2) lineage described in South Africa share 3 mutation positions in common in the spike protein $(\mathrm{K} 417 \mathrm{~N} / \mathrm{T}$, E484K, and N501Y). Both the P.1 and the B.1.351 lineage also have the ORF1b deletion (del 3675-3677 SGF) (6).

This is the fourth recent detection of a major international variant following the detection of the United Kingdom 501Y.V1 variant, the South African 501Y.V2 variant, and the Nigerian B.1.525 variant in Guangdong (7-9). The transmissibility and pathogenicity of these mutant variants urgently need further study.

\section{doi: $10.46234 / \mathrm{ccdcw} 2021.110$ \\ \# Corresponding author: Baisheng Li, libsn@126.com.}

\footnotetext{
${ }^{1}$ Guangdong Provincial Center for Disease Control and Prevention, Guangzhou, Guangdong, China; ${ }^{2}$ National Institute for Viral Disease Control and Prevention, China CDC, Beijing, China.
}

Submitted: May 06, 2021; Accepted: May 07, 2021 

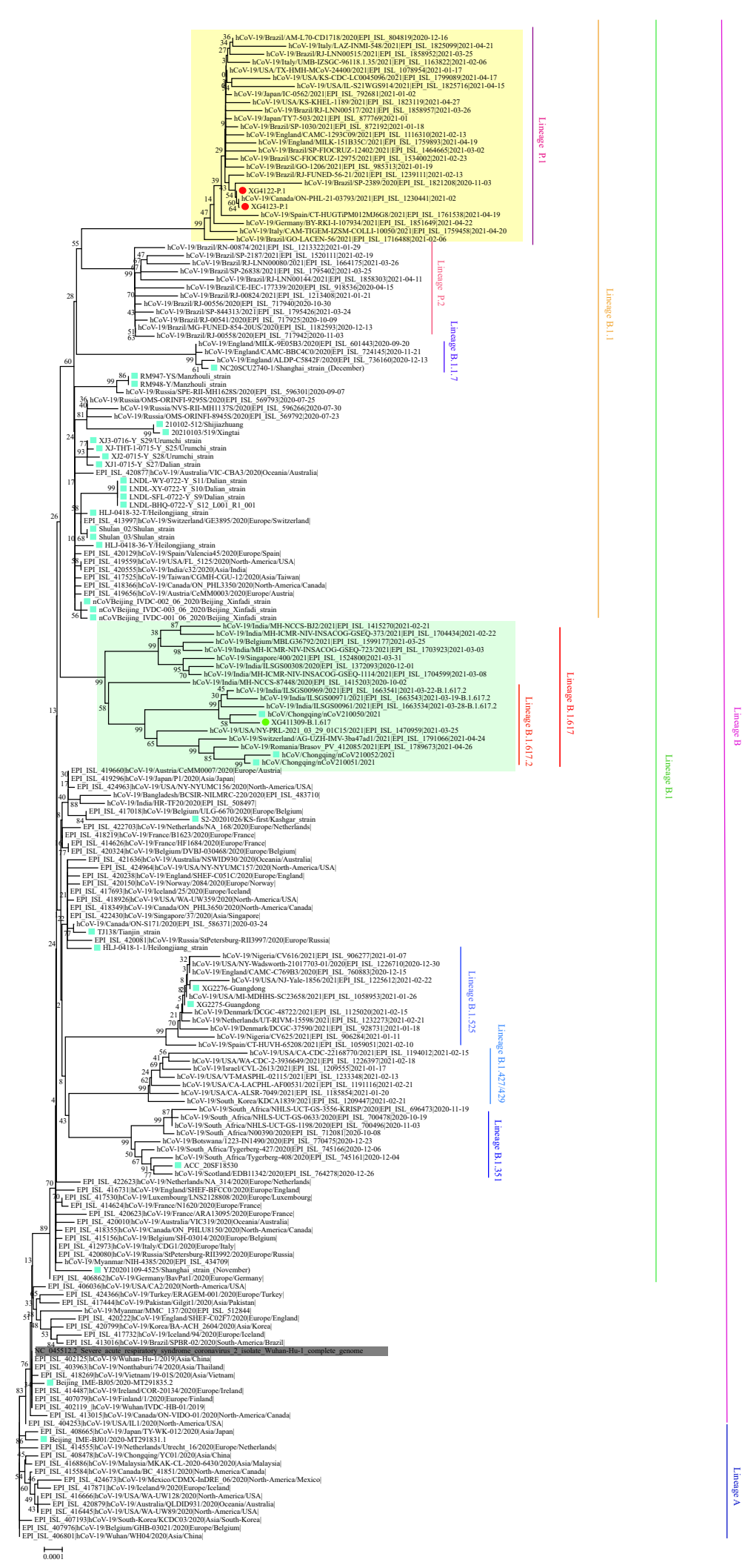

FIGURE 1. Phylogenetic tree based on the full-length genome sequences of the COVID-19 virus. The Brazil variants (P.1) are highlighted in yellow and the Guangdong imported P.1 variants are marked with red dots. The strains associated with specific outbreaks in China are marked with blue squares. The Wuhan reference strain is shaded in gray. The seven distinguished COVID-19 mutants are marked and colored on the right. The A or B lineage of the COVID-19 virus were marked and colored on the right. 


\section{REFERENCES}

1. GISAID. hCoV-19 tracking of variants (see menu option GR/501Y. V3(P.1). https://www.gisaid.org/hcov19-variants/. [2021-4-30].

2. Tan WJ, Zhao X, Ma XJ, Wang WL, Niu PH, Xu WB, et al. A novel coronavirus genome identified in a cluster of pneumonia cases-Wuhan, China 2019-2020. China CDC Wkly 2020;2(4):61 - 2. http://dx. doi.org/10.46234/ccdcw2020.017.

3. Wu F, Zhao S, Yu B, Chen YM, Wang W, Song ZG, et al. A new coronavirus associated with human respiratory disease in China. Nature 2020;579(7798):265. http://dx.doi.org/10.1038/s41586-020-2008-3.

4. Sabino EC, Buss LF, Carvalho MPS, Prete Junior CA, Crispim MA, Fraiji NA, et al. Resurgence of COVID-19 in Manaus, Brazil, despite high seroprevalence. Lancet 2021;397(10273):452 - 5. http://dx.doi.org/ 10.1016/S0140-6736(21)00183-5.

5. Tegally H, Wilkinson E, Giovanetti M, Iranzadeh A, Fonseca V, Giandhari J, et al. Detection of a SARS-CoV-2 variant of concern in South Africa. Nature 2021;592:438 - 43. http://dx.doi.org/10.1038/ s41586-021-03402-9.

6. Galloway SE, Paul P, MacCannell DR, Johansson MA, Brooks JT, MacNeil A, et al. Emergence of SARS-COV-2 B.1.1.7 lineage - United States, December 29, 2020-January12, 2021. https://www.cdc.gov/ mmwr/volumes/70/wr/pdfs/mm7003e2-H.pdf. [2021-1-15].

7. Chen HY, Huang XY, Zhao X, Song Y, Hao P, Jiang H, et al. The first case of new variant COVID-19 originating in the United Kingdom detected in a returning student-Shanghai Municipality, China, December 14, 2020. China CDC Wkly 2021;3(1):1 - 3. http://dx. doi.org/10.46234/ccdcw2020.270.

8. Chen FJ, Li BS, Hao P, Song Y, Xu WB, Liu NK, et al. A case of new variant COVID-19 first emerging in South Africa detected in airplane pilot - Guangdong Province, China, January 6, 2021. China CDC Wkly 2021;3(2):28 - 9. http://dx.doi.org/10.46234/ccdcw2021.007.

9. Hu Y, Zhao X, Li ZC, Kang M, Deng XL, Li BS. Two imported cases of new variant COVID-19 first emerging in Nigeria - Guangdong Province, China, March 12, 2021. China CDC Wkly 2021;3(19):411 3. http://dx.doi.org/10.46234/ccdcw2021.074. 